\title{
CONCERNING PRODUCT INTEGRALS AND EXPONENTIALS
}

\author{
W. P. DAVIS AND J. A. CHATFIELD
}

Abstract. Suppose $S$ is a linearly ordered set, $N$ is the set of real numbers, $G$ is a function from $S \times S$ to $N$, and all integrals are of the subdivision-refinement type. We show that if $\int_{a}^{b} G^{2}=0$ and either integral exists, then the other exists and ${ }_{a} \prod^{b}(1+G)=\exp \int_{a}^{b} G$. We also show that the bounded variation of $G$ is neither necessary nor sufficient for $\int_{a}^{b} G^{2}$ to be zero.

B. W. Helton, J. S. MacNerney, and H. S. Wall have established various relationships between integral equations, sum integrals, and product integrals. This paper establishes a relationship between exponentials, sum integrals, and product integrals which may be used to evaluate certain product integrals or sum integrals. Integrals used are of the subdivision-refinement type and complete definitions of these and other terms and symbols used in this paper may be found in [1] or [2]. Suppose $S$ is a linearly ordered set [2] and $N$ is the set of real numbers. All functions considered will be functions from $S \times S$ to $N$ unless otherwise noted. In [1, Theorem 3.4] it is shown that for functions of bounded variation from $S \times S$ to $N$ the following two statements are equivalent: (1) $\int_{a}^{b} G$ exists and (2) ${ }_{a} \Pi^{b}(1+G)$ exists. Under the hypothesis that $\int_{a}^{b} G^{2}=0$, we show that the following two statements are equivalent for functions from $S \times S$ to $N$ : (1) $\int_{a}^{b} G$ exists and (2) ${ }_{a} \Pi^{b}(1+G)$ exists and is not zero. It is also noted that neither of the following two statements is a consequence of the other. (1) $\int_{a}^{b} G^{2}=0$ and (2) $G$ is of bounded variation on $[a, b]$.

Theorem 0. If $\prod^{b}(1+G)$ exists and is not zero then if $\epsilon>0$ there is a subdivision $D$ of $\{a, b\}$ such that if $D^{\prime}=\left\{x_{i}\right\}_{i=0}^{n}$ is a refinement of $D$, then

$$
\left|\log \frac{{ }_{a} \prod^{b}(1+G)}{\prod_{D^{\prime}}\left(1+G_{i}\right)}\right|<\epsilon .
$$

The proof of this theorem is omitted.

THEOREM 1. Neither of the following statements is a consequence of the other:

Received by the editors December 11, 1969.

AMS Subject Classifications. Primary 2645, 2649; Secondary 4513.

Key Words and Phrases. Exponentials, product integrals, subdivision-refinement type integrals, bounded variation. 
(1) $\int_{a}^{b} G^{2}=0$.

(2) $G$ is of bounded variation.

INDICATION OF PROOF. Let $G$ be the function such that for each $0 \leqq x \leqq 1,0 \leqq y \leqq 1$,

$$
\begin{aligned}
G(x, y) & =x, \quad x=1 / n, \quad n \text { an integer, and }|x-y| \geqq 1 / n-1 /(n+1), \\
& =0, \quad \text { otherwise. }
\end{aligned}
$$

$\int_{0}^{1} G^{2}=0$ but $G$ is not of bounded variation on $[0,1]$ and $\int_{0}^{1} G$ does not exist. Hence (2) is not a consequence of (1).

Let $H$ be the function such that for each $0 \leqq x \leqq 1,0 \leqq y \leqq 1$,

$$
\begin{aligned}
H(x, y) & =1, \quad x=0, \quad y>x, \\
& =0, \quad \text { otherwise. }
\end{aligned}
$$

$V_{0}^{1} H=1$ but $\int_{0}^{1} H^{2}=1$. Hence (1) does not follow from (2).

The following theorem may be found in $[2$, p. 151] and may be established by induction.

THEOREM 2. If $n$ is an integer greater than 1 and each of $\left\{A_{i}\right\}_{i=1}^{n}$ and $\left\{B_{i}\right\}_{i=1}^{n}$ is a sequence of numbers, then

$$
\prod_{i=1}^{n} A_{i}-\prod_{i=1}^{n} B_{i}=\sum_{i=1}^{n}\left(\prod_{j=1}^{i-1} B_{j}\right)\left(A_{i}-B_{i}\right)\left(\prod_{k=i+1}^{n} A_{k}\right)
$$

ThEOREM 3. If $\int_{a}^{b} G^{2}=0$, then the following two statements are equivalent: (1) $\int_{a}^{b} G$ exists.

(2) ${ }_{a} \prod^{b}(1+G)$ exists and is not zero. Furthermore, if either (1) or (2) is true, then $\int_{a}^{b} G=\log _{a} \prod^{b}(1+G)$.

Proof. 1. Suppose (1) is true and $\epsilon>0$. Since $\int_{a}^{b} G^{2}=0$ and $\int_{a}^{b} G$ exist then there is a subdivision $D$ of $\{a, b\}$ such that if $D^{\prime}$ is a refinement of $D$, then there is a number $k$ such that:

$$
\begin{aligned}
& \sum_{D^{\prime}} G_{i}^{2}<\frac{1}{4} \text { and hence }\left|G_{i}\right|<\frac{1}{2}, \\
& \sum_{D^{\prime}} G_{i}^{2}<\frac{\epsilon}{2 \exp \left(\frac{3}{2}+\int_{a}^{b} G\right)}, \\
& |k|<\frac{\epsilon}{8 \exp \left(\frac{3}{2}+\int_{a}^{b} G\right)},
\end{aligned}
$$


(4) $|k|<\frac{1}{2}$, so if $n>m \geqq 0$,

$$
\exp (m k / n)<\exp \left(\frac{1}{2}\right) \quad \text { and } \exp (-k)<\exp \left(\frac{1}{2}\right)
$$

and

$$
\int_{a}^{b} G=\sum_{D^{\prime}} G_{i}+k
$$

Let $D^{\prime}=\left\{x_{i}\right\}_{1=0}^{n}$ be a refinement of $D$.

$$
\begin{aligned}
\sum_{i=1}^{n} \mid \exp & \left(G_{i}+\frac{k}{n}\right)-G_{i}-1 \mid \\
& =\sum_{i=1}^{n}\left|-1-G_{i}+\sum_{j=0}^{\infty} \frac{\left(G_{i}+k / n\right)^{j}}{j !}\right| \\
& \leqq \sum_{i=1}^{n}\left|\frac{k}{n}\right|+\sum_{i=1}^{n}\left|\sum_{j=2}^{\infty} \frac{\left(G_{i}+k / n\right)^{j}}{j !}\right| \\
& \leqq|k|+\sum_{i=1}^{n}\left(G_{i}+k / n\right)^{2} \cdot\left(\sum_{j=2}^{\infty} \frac{1}{j !}\right) \\
& <|k|+\sum_{i=1}^{n}\left(G_{i}+k / n\right)^{2} \\
& \leqq|k|+\frac{\epsilon}{2 \exp \left(\frac{3}{2}+\int_{a}^{b} G\right)}+|k|+|k| \\
& <\frac{8 \exp \left(\frac{3}{2}+\int_{a}^{b} G\right)}{}
\end{aligned}
$$

Therefore,

$$
\begin{array}{r}
\sum_{i=1}^{n}\left|\exp \left(G_{i}+k / n\right)-G_{i}-1\right| \\
<\frac{7 \epsilon}{8 \exp \left(\frac{3}{2}+\int_{a}^{b} G\right)} .
\end{array}
$$

Then, 


$$
\begin{aligned}
& \left|\prod_{i=1}^{n}\left(1+G_{i}\right)-\exp \left(\int_{a}^{b} G\right)\right| \\
& =\left|\prod_{i=1}^{n}\left(1+G_{i}\right)-\prod_{i=1}^{n} \exp \left(G_{i}+k / n\right)\right| \\
& \leqq \sum_{i=1}^{n}\left|\prod_{j=1}^{i-1}\left(1+G_{i}\right)\right| \cdot\left|\exp \left(G_{i}+k / n\right)-1-G_{i}\right| \cdot\left|\prod_{j=i+1}^{n} \exp \left(G_{i}+k / n\right)\right| \\
& \leqq \sum_{i=1}^{n}\left|\prod_{j=1}^{i-1} \exp G_{i}\right| \cdot\left|\prod_{j=i+1}^{n} \exp \left(G_{i}+k / n\right)\right| \cdot\left|\exp \left(G_{i}+k / n\right)-1-G_{i}\right| \\
& =\sum_{i=1}^{n}\left|\exp \left(\sum_{j=1}^{n} G_{j}+k-G_{i}-k+((n-i) / n) k\right)\right| \\
& <\sum_{i=1}^{n} \exp \left(\int_{a}^{b} G\right) \cdot \exp \left(\frac{1}{2}\right) \cdot \exp \left(\frac{1}{2}\right) \cdot \exp \left(\frac{1}{2}\right) \cdot\left|\exp \left(G_{i}+k / n\right)-1-G_{i}\right| \\
& <\exp \left(\int_{a}^{b} G+\frac{3}{2}\right) \frac{7 \epsilon}{8 \exp \left(\int_{a}^{b} G+\frac{3}{2}\right)}
\end{aligned}
$$

$<\epsilon$.

Hence, $\left|\prod_{i=1}^{n}\left(1+G_{i}\right)-\exp \left(\int_{a}^{b} G\right)\right|<\epsilon$ so that ${ }_{a} \prod^{b}(1+G)$ exists and is $\exp \left(\int_{a}^{b} G\right)$.

2. Suppose (2) is true and $\epsilon>0$. Since $\int_{a}^{b} G^{2}=0,{ }_{a} \prod^{b}(1+G)$ exists and is not zero, then there exists a subdivision $D$ of $\{a, b\}$ such that if $D^{\prime}$ is a refinement of $D$, then

$$
\begin{gathered}
\left|G_{i}\right|<\frac{1}{2} \\
\left|\log \frac{\prod^{b}(1+G)}{\prod_{D^{\prime}}\left(1+G_{i}\right)}\right|<\frac{\epsilon}{2} \\
\log \left(1+G_{i}\right)=\sum_{j=1}^{\infty} \frac{(-1)^{j-1} G_{i}^{j}}{j} \\
M=\sum_{j=2}^{\infty} \frac{\left(\frac{1}{2}\right)^{j-2}}{j} \geqq \sum_{j=2}^{\infty} \frac{\left|\left(G_{i}\right)^{j-2}\right|}{j} \\
\sum_{D^{\prime}} G_{i}^{2}<\frac{\epsilon}{2 M} .
\end{gathered}
$$

Let $D^{\prime}=\left\{x_{i}\right\}_{i=0}^{n}$ be a refinement of $D$, then 


$$
\begin{aligned}
\mid \log _{a} \Pi^{b}(1+G) & -\sum_{i=1}^{n} G_{i} \mid \\
& \leqq\left|\log \prod_{i=1}^{n}\left(1+G_{i}\right)-\sum_{i=1}^{n} G_{i}\right|+\left|\log \frac{a \prod^{b}(1+G)}{D^{\prime} \Pi\left(1+G_{i}\right)}\right| \\
& <\left|\sum_{i=1}^{n}\left[\log \left(1+G_{i}\right)-G_{i}\right]\right|+\frac{\epsilon}{2} \\
& =\left|\sum_{i=1}^{n}\left[\sum_{j=1}^{\infty}(-1) \frac{G_{i}{ }^{j}}{j}-G_{i}\right]\right|+\frac{\epsilon}{2} \\
& =\left|\sum_{i=1}^{n} \sum_{j=2}^{\infty}(-1) \frac{{ }^{j-1} G_{i}^{j}}{j}\right|+\frac{\epsilon}{2} \\
& =\left|\sum_{i=1}^{n}\left[G_{i}^{2} \cdot \sum_{j=2}^{\infty}(-1)^{j-1} \frac{G_{i}^{j}}{j}\right]\right|+\frac{\epsilon}{2} \\
& \leqq \sum_{i=1}^{n}\left[G_{i}^{2} \cdot \sum_{j=2}^{\infty} \frac{\left|G_{i}\right|{ }^{j-2}}{j}\right]+\frac{\epsilon}{2} \\
& \leqq M \sum_{i=1}^{n} G_{i}^{2}+\frac{\epsilon}{2}<M \cdot \frac{\epsilon}{2 M}+\frac{\epsilon}{2}=\epsilon .
\end{aligned}
$$

Hence,

$$
\left|\log _{a} \Pi^{b}(1+G)-\sum_{i=1}^{n} G_{i}\right|<\epsilon
$$

so that $\int_{a}^{b} G$ exists and is $\log _{a} \prod^{b}(1+G)$.

Remark. As noted by the referee, a function $G$ from $S \times S$ to $N$ may have the property that $\int_{a}^{b} G^{2}=0$ and $\int_{a}^{b} G$ exists yet $G$ fails to be of bounded variation on $[a, b]$. As an example of such a function we offer the following: Suppose for $0<x \leqq 1, g(x)=x \sin (\pi / x)$ and $g(0)=0$ and for each $0 \leqq x \leqq 1,0 \leqq y \leqq 1, G(x, y)=g(y)-g(x) . \int_{0}^{1} G^{2}$ $=\int_{0}^{1} G=0$, but $\int_{0}^{1}|G|$ does not exist.

\section{BIBLIOGRAPHY}

1. B. W. Helton, Integral equations and product integrals, Pacific J. Math. 16 (1966), 297-322. MR $32 \# 6167$.

2. J. S. MacNerney, Integral equations and semigroups, Illinois J. Math. 7 (1963), 148-173. MR $26 \# 1726$.

3. H. S. Wall, Concerning harmonic matrices, Arch. Math. 5 (1954), 160-167. MR $15,801$.

Southwest Texas State University, San Marcos, Texas 78666 